\title{
A remark on the multiplicity of monotone periodic orbits
}

\author{
GLEN R. HALL \\ Department of Mathematics, Boston University, 111 Cummington Street, \\ Boston, MA 02215, USA
}

\begin{abstract}
We give a new proof of the fact that an area-preserving monotone twist map of the annulus with one $p / q$-periodic orbit on which the map preserves the ordering on the angular coordinate (i.e. Birkhoff or monotone periodic orbits) actually has a second such orbit distinct from the first.
\end{abstract}

\section{Introduction}

In this note we give a new proof of the following:

THEOREM 1 ([3], [10] and [11]). If $f$ is an area-preserving monotone twist map of an annulus onto itself with a monotone periodic orbit of rotation number $p / q$, then $f$ actually has two distinct monotone periodic orbits of rotation number $p / q$.

The hope is to give a proof of the above theorem which will generalize to higher-dimensional analogues of area-preserving monotone twist maps. The proof we give here relies on the classical theorem of Birkhoff (see [4] and particularly [5]), which states that an area-preserving twist map of the annulus actually has two distinct periodic orbits for any possible rotation number. This theorem has a higher-dimensional analogue recently proven by Conley and Zehnder [7].

The other ingredient in theorem 1 is that monotone and non-monotone orbits for annulus maps have very distinct topological characteristics, i.e. they can be isolated from each other in a strong sense. This allows one to build a modification of a given area-preserving monotone twist map which destroys all non-monotone periodic orbits, but which does not introduce any new periodic orbits of the given rotation number (see also [2]). Then Birkhoff's theorem implies that the modified map has two distinct and necessarily monotone orbits which must also be orbits of the original map. What is missing for the higher-dimensional versions is a definition of 'monotone' or ordered orbits which allows this sort of isolation from non-ordered orbits.

The above theorem 1 can be used as the crucial step in a proof of the theorem of Aubry [3] and Mather [11] which states that all area-preserving monotone twist maps have orbits of all possible rotation numbers on which the angular order is preserved. We give an outline of this proof in the final section. It is very similar to the proof of the Aubry-Mather theorem given in [8]. 


\section{Notations and definitions}

We let $A=\{(x, y): x \in \mathbf{R}, 0 \leq y \leq 1\}$ be the strip, the universal cover of the annulus; we let

$$
\left.\left.\begin{array}{l}
\pi_{1} \\
\pi_{2}
\end{array}\right\}: A \rightarrow \mathbf{R} \quad \text { and } \quad \begin{array}{l}
\pi_{1} \\
\pi_{2} \\
\pi_{3}
\end{array}\right\}: A \times \mathbf{R} \rightarrow \mathbf{R}
$$

be the usual projections; and we make the following:

Definition. An area-preserving twist map is a diffeomorphism $f: A \rightarrow A$ which satisfies:

(1) $f$ preserves orientation, boundary components and area;

(2) $\forall(x, y) \in A, f(x+1, y)=f(x, y)+(1,0)$;

(3) the rotation number of $\left.f\right|_{\{(x, 0)\}}<$ rotation number of $\left.f\right|_{\{(x, 1)\}}$.

Also, $f$ is called an area-preserving monotone twist map if $f$ satisfies (1), (2) above and

$$
\forall(x, y) \in A, \quad \frac{\partial \pi_{1} \circ f}{\partial y}(x, y)>0 .
$$

Finally, a point $z \in A$ is called a $p / q$-periodic point if $p, q$ are relatively prime and $f^{q}(z)=z+(p, 0)$.

We recall the theorem of Birkhoff.

THEOREM 2 ([4] and [5]). If $f: A \rightarrow A$ is an area-preserving twist map with rotation number of $\left.f\right|_{\{(x, 0)\}} \leq p / q \leq$ rotation number of $\left.f\right|_{\{(x, 1)\}}$, then $f$ has at least two distinct $p / q$-periodic orbits.

Remark. We will use the fact that this holds for continuous maps.

We will also use the following theorem of Moser [12] which allows us to suspend a given area-preserving monotone twist map in a very useful way, i.e. we can give the map as the time one map of a non-autonomous Hamiltonian system or a flow on $\boldsymbol{A} \times \mathbf{R}$, the extra variable denoting time.

TheOREM 3 [12]. If $f: A \rightarrow A$ is an area-preserving monotone twist map, then there exists a function $H: A \times \mathbf{R} \rightarrow \mathbf{R}$ such that $H$ is periodic of period 1 in $x$ and $t, \partial^{2} H / \partial y^{2}>0$ and if $\phi: A \times \mathbf{R} \times \mathbf{R} \rightarrow \boldsymbol{A} \times \mathbf{R}$ is the solution of the Hamiltonian system

$$
\frac{d x}{d s}=\frac{\partial H}{\partial y}(x, y, t), \quad \frac{d y}{d s}=-\frac{\partial H}{\partial x}(x, y, t), \quad \frac{d t}{d s}=1
$$

satisfying $\phi(z, t, 0)=(z, t)$ for all $(z, t) \in A \times \mathbf{R}$, then $f(z)=\pi_{1}(\phi(z, 0,1))$, $\pi_{2}(\phi(z, 0,1))$. (That is, any area-preserving monotone twist map is the time one map of a time periodic Hamiltonian system satisfying a Legendre condition.)

Remark. We will prove theorem 1 for maps which arise as the time one map of $C^{2}$ Hamiltonians as above.

From now on we think of $f: A \rightarrow A$ as a given area-preserving monotone twist map with associated flow $\phi$ on $A \times \mathbf{R}$. We can use this flow to help describe the topological nature of periodic orbits of $f$. 
Definition. A $p / q$-periodic point $z \in A$ is called monotone (or Birkhoff) if for any $w_{1}, w_{2} \in\left\{f^{i}(z)+(j, 0): i, j\right.$ in $\left.\mathbf{Z}\right\}$,

$$
\pi_{1}\left(w_{1}\right)<\pi_{1}\left(w_{2}\right) \text { implies } \pi_{1}\left(f\left(w_{1}\right)\right)<\pi_{1}\left(f\left(w_{2}\right)\right) .
$$

The condition on the second partial of $H$ with respect to $y$ implies that for a monotone orbit $\pi_{1}\left(w_{1}\right)<\pi_{1}\left(w_{2}\right)$ implies $\pi_{1}\left(\phi\left(w_{1}, 0, t\right)\right)<\pi_{1}\left(\phi\left(w_{2}, 0, t\right)\right)$ for all $t$. This follows from the Legendre condition $\partial^{2} H / \partial^{2} y>0$, which implies that $d x / d s$ increases as $y$ increases. So if one orbit 'passes' the other in the $x$ direction, the 'faster' orbit must have the larger $y$ value. Hence we may informally say that a periodic orbit is monotone if the braid associated with it by the flow $\phi$ is trivial. Notice that the monotone twist condition implies that if this braid is non-trivial then it must be 'positive', i.e. any two strands wrap in the same direction (see [8] and figure 1).
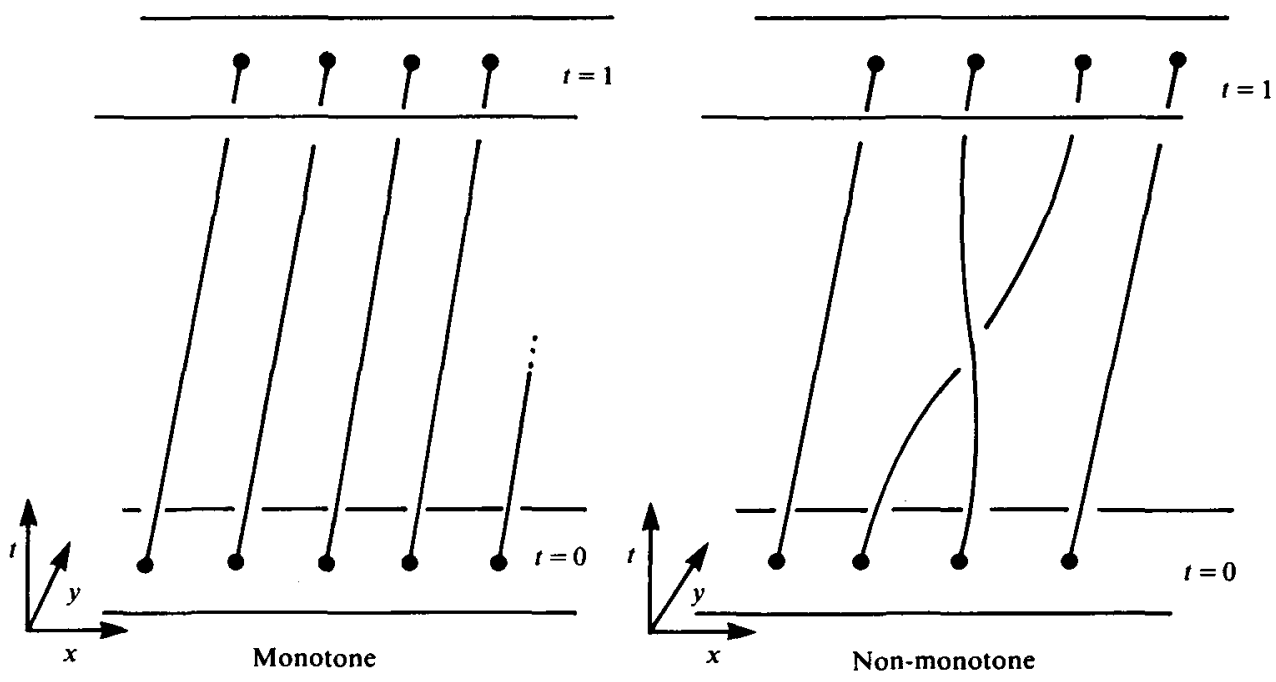

FIGURE 1

The following easy lemma is crucial to the proof of theorem 1.

LEMMA. Suppose $f: A \rightarrow A$ is an area-preserving monotone twist map with $p / q$-monotone periodic point $z$ and $p / q$-non-monotone periodic point $w$. Then there exist $z_{1} \in$ $\left\{f^{i}(z)+(j, 0): i, j \in \mathbf{Z}\right\}$ and $w_{1} \in\left\{f^{i}(w)+(j, 0): i, j \in \mathbf{Z}\right\}$ such that $\pi_{1}\left(z_{1}\right)<\pi_{1}\left(w_{1}\right)$ but $\pi_{1}\left(f\left(z_{1}\right)\right) \geq \pi_{1}\left(f\left(w_{1}\right)\right.$ ) (i.e. the braid given by the $\phi$ orbit of $z$ must link with the braid given by the $\phi$ orbit of $w)$.

Proof of the lemma. Suppose not. Then the monotone twist condition implies that $\pi_{1}\left(z_{1}\right) \neq \pi_{1}\left(w_{1}\right)$ for all $z_{1} \in\left\{f^{i}(z)+(j, 0): i, j \in \mathbf{Z}\right\}$ and $w_{1} \in\left\{f^{i}(w)+(j, 0): i, j \in \mathbf{Z}\right\}$. Moreover, for any such $w_{1}$ there exists $z_{1}, z_{2} \in\left\{f^{i}(z)+(j, 0): i, j \in \mathbf{Z}\right\}$ with $\pi_{1}\left(z_{1}\right)<$ $\pi_{1}\left(w_{1}\right)<\pi_{1}\left(z_{2}\right)$ and no point $z_{3} \in\left\{f^{i}(z)+(j, 0): i, j \in \mathbf{Z}\right\}$ with $\pi_{1}\left(z_{1}\right)<\pi_{1}\left(z_{3}\right)<$ $\pi_{1}\left(z_{2}\right)$. But then $\pi_{1}\left(f^{i}\left(z_{1}\right)\right)<\pi_{1}\left(f^{i}\left(w_{1}\right)\right)<\pi_{1}\left(f^{i}\left(z_{2}\right)\right)$ for all $i$ and, since the sets $\left\{f^{i}(z)+(j, 0): i, j \in \mathbf{Z}\right\}$ and $\left\{f^{i}(w)+(j, 0): i, j \in \mathbf{Z}\right\}$ both have exactly $q$ elements with 
$x$ coordinates in $[0,1)$, we see that $w$ must be monotone. But this contradicts the assumption that $w$ is non-monotone, hence the proof is complete.

Proof of theorem 1. Fix an area-preserving monotone twist map $f$ with associated Hamiltonian $H$ and Hamiltonian flow $\phi$ as above. We assume that $z_{0} \in A$ is a $p / q$-monotone periodic point for $f$ and we must shpw that $f$ has another $p / q$ monotone periodic point not on the orbit of $z_{0}$.

For each point $(z, t) \in A \times \mathbf{R}$ and $\varepsilon>0$ we let

$$
C^{+}(z, t, \varepsilon)=\left\{(w, t):\left(\pi_{2}(w)-\pi_{2}(z)\right)>(1 / \varepsilon)\left|\pi_{1}(w)-\pi_{1}(z)\right|\right\}
$$

and

$$
C^{-}(z, t, \varepsilon)=\left\{(w, t):\left(\pi_{2}(z)-\pi_{2}(w)\right)>(1 / \varepsilon)\left|\pi_{1}(w)-\pi_{1}(z)\right|\right\}
$$

(see figure 2). Note that when $\varepsilon$ is sufficiently small, the left boundary of $C^{+}(z, t, \varepsilon) \sim$ $(z, t)$ and the right boundary of $C^{-}(z, t, \varepsilon) \sim(z, t)$ are entrance set, while the right boundary of $C^{+}(z, t, \varepsilon) \sim(z, t)$ and the left boundary of $C^{-}(z, t, \varepsilon) \sim(z, t)$ are exit set for $\phi$ (see [6]).

$$
t=1
$$
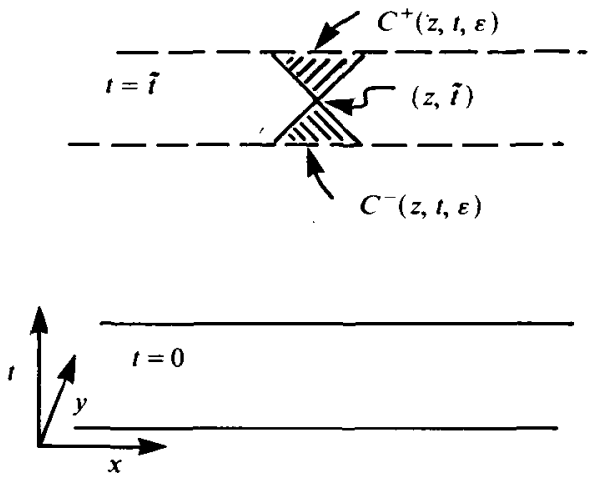

Figure 2

The idea of the proof is to construct a new area-preserving twist map $g: A \rightarrow A$ which agrees with $f$ on a large set including the orbit of $z_{0}$, which has no $p / q$ monotone periodic orbits except those of $f$ and such that $g$ has no $p / q$-non-monotone periodic orbits. The map $g$ will be far from $f$-in particular $g$ will not be a monotone twist map. However, $g$ will still be a twist map and will still be the time one map of a Hamiltonian flow, so it will be area-preserving. We construct $g$ by altering the Hamiltonian $H$ on the sets $C^{+}(\phi(z, 0, t), \varepsilon)$. In particular we alter $H$ so that for most of the flow the set $\left\{(w, t): \pi_{1}(w)=\pi_{1}\left(\phi\left(z_{0}, 0, t\right)\right)\right\}$ is invariant; hence no orbits will be able to link with the orbit of $z_{0}$. 
Fix $K$ larger than the $C^{2}$ norm of $H$. Then we define a function $\tilde{H}_{\varepsilon}$ on the cone in $\mathbf{R}^{2}$ as follows:

$$
\begin{gathered}
\tilde{H}_{\varepsilon}:\{(x, y): \varepsilon y \geq|x| \text { or }-\varepsilon y \geq|x|\} \rightarrow \mathbf{R} \\
\tilde{H}_{\varepsilon}:(x, y) \rightarrow\left(4 K / \varepsilon^{2}\right) x^{2} .
\end{gathered}
$$

The level sets of $\tilde{H}_{\varepsilon}$ are pictured in figure 3 . In particular we notice that $\partial \tilde{H}_{\varepsilon} / \partial x<0$ for $x<0, \partial \tilde{H}_{\varepsilon} / \partial x>0$ for $x>0$ and $\tilde{H}_{\varepsilon}(0, y)=0$ for all $y$, so the Hamiltonian flow for $\tilde{H}_{\varepsilon}$ will flow up on the left of $x=0$ and down on the right.

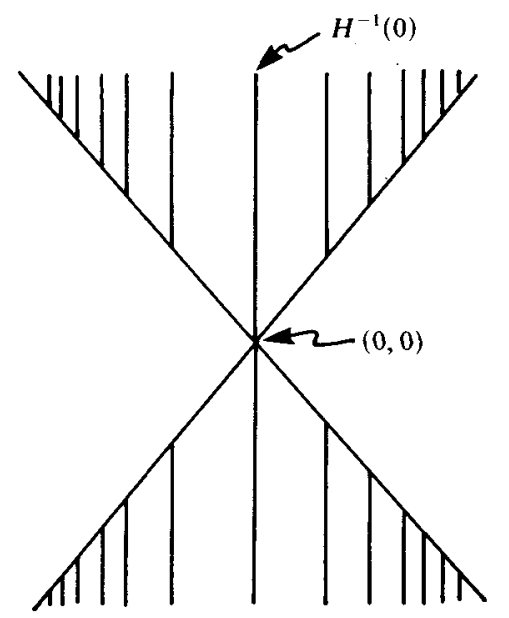

Figure 3

Let $\psi_{1}$ be a bump function as pictured in figure 4. For each $t \in[0, q]$ let $\left\langle\phi\left(z_{0}, 0, t\right)\right\rangle=(w, s)$, where $\pi_{1}(w)$ and $s$ are the fractional parts of $\pi_{1}\left(\phi\left(z_{0}, 0, t\right)\right)$ and $t$ respectively and $\pi_{2}(w)=\pi_{2}\left(\phi\left(z_{0}, 0, t\right)\right)$. Hence the set $\left\{\left\langle\phi\left(z_{0}, 0, t\right)\right\rangle: t \in[0, q]\right\}$ is as pictured in figure 5 .

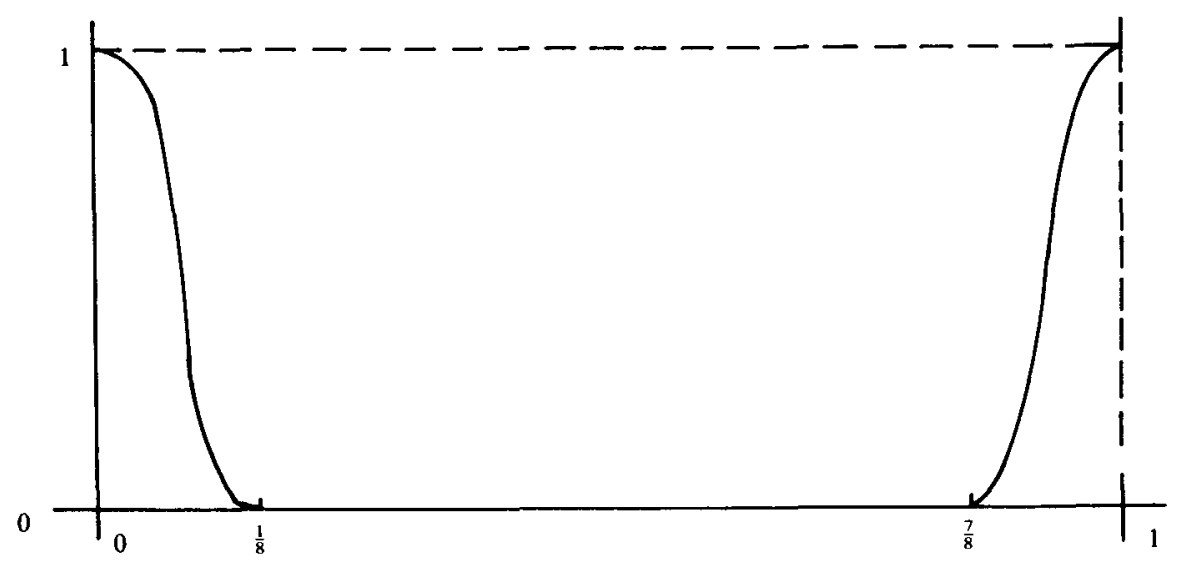

Figure 4 


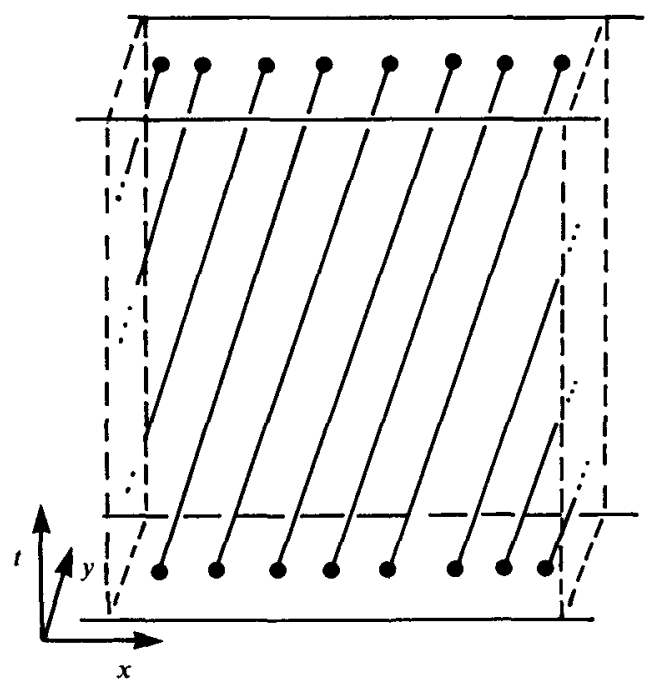

Figure 5

We now define our new Hamiltonian $H_{1, \varepsilon}: A \times \mathbf{R} \rightarrow \mathbf{R}$ for $(w, t) \in$ $[0,1] \times[0,1] \times[0,1]$ by first letting

$$
\tilde{H}_{1, \varepsilon}(w, t)\left\{\begin{aligned}
= & H(w, t) \text { if }(w, t) \notin C^{ \pm}\left(\left\langle\phi\left(z_{0}, 0, t\right)\right\rangle, \varepsilon\right) \text { for } t \in[0, q], \\
= & \left(1-\psi_{1}((x / \varepsilon y+1) / 2)\right)\left(\tilde{H}_{e}(x, y)+H\left(\left\langle\phi\left(z_{0}, 0, t\right)\right\rangle\right)\right. \\
& \left.+(\partial H / \partial x)\left(\left\langle\phi\left(z_{0}, 0, t\right)\right\rangle\right) x+(\partial H / \partial y)\left(\left\langle\phi\left(z_{0}, 0, t\right)\right\rangle\right) y\right) \\
& +\psi_{1}((x / \varepsilon y+1) / 2) \cdot H(w, t) \\
& \text { if }(w, t) \in C^{ \pm}\left(\left\langle\phi\left(z_{0}, 0, t\right)\right\rangle, \varepsilon\right) \text { for } t \in[0, q], \\
& \text { where } x=\pi_{1}(w)-\pi_{1}\left(\left\langle\phi\left(z_{0}, 0, t\right)\right\rangle\right) \text { and } y=\pi_{2}(w)-\pi_{2}\left(\left\langle\phi\left(z_{0}, 0, t\right)\right\rangle\right),
\end{aligned}\right.
$$

i.e. $H$ and $\tilde{H}_{1, \varepsilon}$ agree except in prisms with edge along the orbit of $z_{0}$ under $\phi$ (see figure 6). By the choice of $\tilde{H}_{\varepsilon}$ the orbits of the Hamiltonian flow for $\tilde{H}_{1, \varepsilon}$ which enter $C^{ \pm}\left(\left\langle\phi\left(z_{0}, 0, t\right)\right\rangle, \varepsilon\right)$ will reach $C^{ \pm}\left(\left\langle\phi\left(z_{0}, 0, t\right)\right\rangle, \varepsilon / 2\right)$. The level sets of $\tilde{H}_{1, \varepsilon}$ in a $C^{ \pm}\left(\left\langle\phi\left(z_{0}, 0, t\right)\right\rangle, \varepsilon\right)$ are as pictured in figure 7 . Finally we need to make $\tilde{H}_{1, \varepsilon}$ equal to $H$ again in one of the prisms so that the resulting time $q$ map from this Hamiltonian will have the boundary twist condition, i.e. we let

$$
\tilde{H}_{1, \varepsilon}(w, t)\left\{\begin{aligned}
= & H(w, t) \text { if }(w, t) \notin C^{ \pm}\left(\left\langle\phi\left(z_{0}, 0, t\right)\right\rangle, \varepsilon\right) \text { for } t \in[0,1], \\
= & \left(1-\psi_{1}(t)\right) \tilde{H}_{1, \varepsilon}(w, t)+\psi_{1}(t) \cdot H(w, t) \\
& \text { if }(w, t) \in C^{ \pm}\left(\left\langle\phi\left(z_{0}, 0, t\right)\right\rangle, \varepsilon\right) \text { for } t \in[0,1] .
\end{aligned}\right.
$$

We can extend $H_{1, \varepsilon}$ to all of $A \times \mathbf{R}$ by periodicity in $x$ and $t$.

Let $\Phi_{\varepsilon}: A \times \mathbf{R} \times \mathbf{R} \rightarrow A \times \mathbf{R}$ be the flow generated by the Hamiltonian $H_{1, \varepsilon}$. Away from the $\phi$ orbit of $z_{0}, H_{1, \varepsilon}$ is $C^{\infty}$ so $\Phi_{\varepsilon}$ is well defined and area-preserving (i.e. for any set not containing a point on the orbit of $z_{0}, \Phi_{\varepsilon}$ preserves the area), and the vector field given by $H_{1, \varepsilon}$ is continuous along the orbit of $z_{0}$ so the resulting time one map is continuous. (Since the vector field is only $C^{1}$ at $z_{0}$, we need to worry about the uniqueness of solutions; however, this follows from the definition 


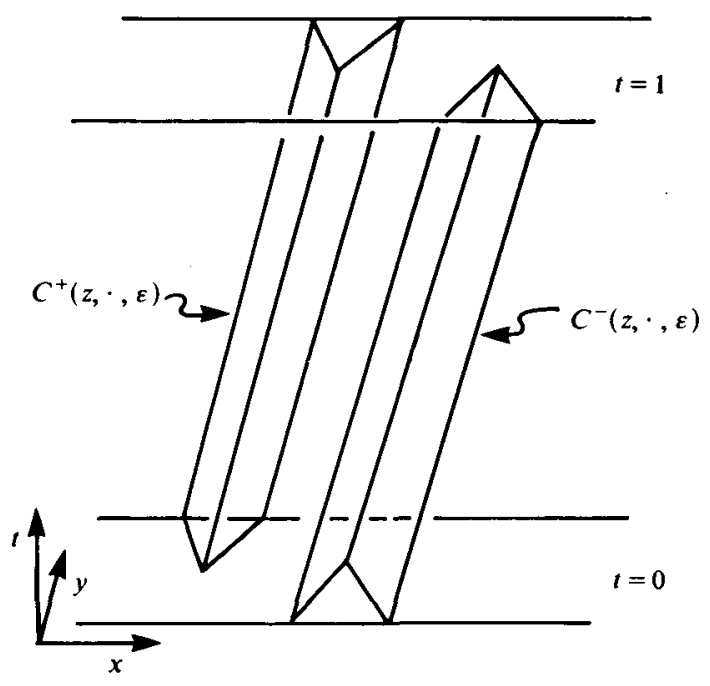

Figure 6

of $H_{1, \varepsilon}$ since no flow lines of $\Phi_{\varepsilon}$ hit the orbit of $z_{0}$ by construction. In fact, the vector field given by $H_{1, \varepsilon}$ is Lipschitz.) We may fix $\varepsilon>0$ so small that if $(w, 0) \in$ $C^{ \pm}\left(z_{0}, 0, \varepsilon\right) \sim\left(z_{0}, 0\right)$ then $\Phi_{\varepsilon}(w, 0,1) \notin C^{ \pm}\left(\phi\left(z_{0}, 0,1\right), \varepsilon\right)$. This follows from the fact that $\partial^{2} H / \partial y^{2}>0$ (i.e. the monotone twist condition for $f$ ) and the fact that $H$ and $H_{1, \varepsilon}$ agree on $C^{ \pm}\left(\left\langle\phi\left(z_{0}, 0, t\right)\right\rangle, \varepsilon\right)$ for $t \in\left[\frac{1}{8}, \frac{7}{8}\right]$. We fix such a small $\varepsilon$, we let $H_{1}=H_{1, \varepsilon}$ and $\Phi=\Phi_{\varepsilon}$ and we define $g(z)=\Phi(z, 0,1)$.

We claim the following:

(1) The orbit of $z_{0}$ under $\Phi$ is the same as the orbit of $z_{0}$ under $\phi$.

(2) The map $g$ has no $p / q$-non-monotone periodic orbits.

(3) Any $p / q$-monotone periodic orbit of $g$ is a $p / q$-monotone periodic orbit of $f$.

(4) The map $g$ has at least two $p / q$-periodic orbits.

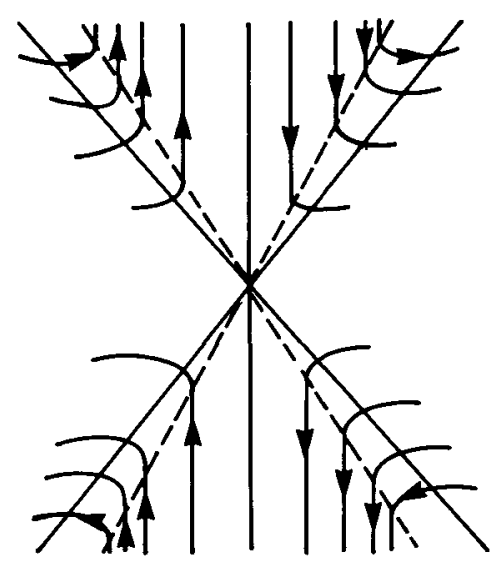

Figure 7 
Claim (1) follows immediately from the construction of $\Phi$. Claim (2) follows from the lemma of the last section which says a non-monotone $p / q$-periodic orbit must link with the orbit of $z_{0}$. The construction of $\Phi$ breaks any $p / q$-periodic orbits of $\phi$ which link with the orbit of $z_{0}$ since no $\Phi$ orbit can pass completely around the $\Phi$ orbit of $z_{0}$. Claim (3) is clear since no $p / q$-periodic orbit of $\Phi$ may enter the set $\bigcup_{t \in[0, q]} C^{ \pm}\left(\left\langle\phi\left(z_{0}, 0, t\right)\right\rangle, \varepsilon\right)$ or any of the translates of this set in $x$, and the flows $\phi$ and $\Phi$ agree off this set. Claim (4) follows from Birkhoff's theorem (theorem 2 above) applied to the twist map $g^{q}$. Note that $g$ will not in general be a monotone twist map since we have not made any effort to control the second partial of $H_{1}$ with respect to $y$; however, by leaving $H$ unaltered on $C^{ \pm}\left(\left\langle\phi\left(z_{0}, 0, t\right)\right\rangle, \varepsilon\right)$ for $t \in\left[\frac{1}{8}, \frac{7}{8}\right]$, we guarantee that the map $g^{q}$ will advance the boundary $y=1$ strictly more to the right than it does the boundary $y=0$. Hence we can apply Birkhoff's theorem.

This completes the proof since the two $p / q$-periodic orbits of $g$ must be $p / q$ Birkhoff periodic orbits and also orbits of $f$. So $f$ has two $p / q$-Birkhoff periodic orbits as we wished to show.

\section{The Aubry-Mather theorem}

Using theorem 1 we can give a proof of the theorem of Aubry and Mather (see $[3],[4]$ and also [10]) similar to the proof in [8].

THEOREM 4 ([3] and [4]). If $f: A \rightarrow A$ is an area-preserving monotone twist map and $\alpha \in \mathbf{R}$ is between the rotation numbers of $f$ on the inner and outer boundary components of $A$, then there is a point $z \in A$ such that $f$ restricted to the set $\left\{f^{i}(z)+(j, 0): i, j \in \mathbf{Z}\right\}$ preserves the ordering in the $x$ coordinate and the rotation number of $f$ at $z$ is $\alpha$.

Proof. When $\alpha=p / q$ the point $z$ may be chosen to be a $p / q$-monotone periodic point, and when $\alpha \notin \mathbf{Q}$ we obtain a minimal set on which $f$ preserves the ordering of the $x$ coordinate with rotation number $\alpha$.

Katok [10] has shown that the orbits on which the $x$ coordinate ordering is preserved by $f$ may be used in limit procedures to obtain other such orbits, and that the rotation number function behaves continuously on these orbits; hence it suffices for us to prove theorem 4 for $\alpha=p / q$ rational. So fix a rational $p / q$.

Given an area-preserving monotone twist map $f$ with associated Hamiltonian $H$, we let $H_{s}, s \in[0,1]$ be a one-parameter family of Hamiltonians periodic in $x$ and $t$ with $H_{1}=H$ and $H_{0}(x, y, t)=\frac{1}{2} c y^{2}$ for some constant $c$ with the time one map of $H_{s}$ having $p / q$-periodic points for all $s \in[0,1]$. For $s$ sufficiently small the $p / q$ periodic points of $f_{s}$ will all be monotone, and it follows easily from arguments of Katok [10] that the set $s$ where $f_{s}$ has a $p / q$-monotone periodic orbit is a closed set in $[0,1]$. We claim that the set $s \in[0,1]$ where $f_{s}$ has a $p / q$-monotone periodic orbit is also open. Suppose $s_{0}$ is on the boundary of this set. Then $f_{s_{0}}$ has $p / q$ monotone periodic orbits. If any of these orbits, say the orbit of $z_{0}$, is isolated, then we may apply theorem 1 , using it to obtain a map having no $p / q$-non-monotone periodic orbits. Away from the orbit of $z_{0}$ we may perturb this map to eliminate all 
other $p / q$-monotone orbits by replacing $H_{s_{0}}$ by $H_{s_{0}+\varepsilon}$. This gives a twist map with only one $p / q$-periodic orbit, contradicting Birkhoff's theorem.

If $f_{s_{0}}$ has no isolated $p / q$-monotone periodic points, then we show that the set of monotone $p / q$-periodic points is fairly well behaved. Again, as above, fix a $p / q$ monotone periodic point $z_{0}$ for $f_{s_{0}}$ and repeat the construction above, creating a new area-preserving twist map $g$ with the property that no $p / q$-periodic orbit has a suspension which links with the suspension of the oribt of $z_{0}$. If $z_{0}$ is not an isolated $p / q$-periodic point for $g$, then all the $p / q$-periodic points of $f_{s_{0}}$ sufficiently close to $z_{0}$ have orbits under the suspension of $f_{s_{0}}$ which do not link with the suspension of the orbit of $z_{0}$. We claim that the $p / q$-periodic points in a neighbourhood of $z_{0}$ will therefore lie on the graph of a smooth function. To see this we note that $(\partial / \partial y)\left(\pi_{1} f_{s_{0}}^{q}\left(z_{0}\right)\right) \neq 0$, so applying the implicit function theorem to the equation $\pi_{1}\left(f_{s_{0}}^{q}(z)\right)-\pi_{1}(z)-p=0$ we see the points satisfying this equation near $z_{0}$ lie on the graph of a smooth function $y=\psi(x)$.

Next we note that if the set of $p / q$-periodic points separates $A$, then this set actually lies on the graph of a smooth function. (That it lies on the graph of a Lipschitz function follows from a theorem of Birkhoff (see Fathi in [9]); that the graph is actually smooth follows from the preceding paragraph.) In this case $f_{s_{0}}$ could have no non-monotone $p / q$-periodic points since the suspension of such an invariant circle of points would separate $A \times \mathbf{R}$ so no $p / q$-periodic orbit could link with any other.

In the case that the $p / q$-monotone periodic points do not separate $A$, we may surround $z_{0}$ with a neighbourhood such that $g$ has no $p / q$-periodic points on the boundary of the neighbourhood and all the $p / q$-periodic points of $g$ lie on the graph of a smooth function $y=\psi(x)$, where $\pi_{1}\left(f_{s_{0}}^{q}(x, \psi(x))\right)-x-p=0$. Since for any $\varepsilon>0$ sufficiently small we have assumed that $f_{s_{0}+\varepsilon}$ has no monotone $p / q$-periodic points, we see that the image of the graph of $\psi$ under $f_{s_{0}}^{q}(\cdot)-(p, 0)$ cannot cross itself transversally (or even topologically transversally) but must lie either above or below the graph of $\psi$ with points of tangency at the $p / q$-monotone periodic points (see figure 8).

By perturbing the Hamiltonian within the shaded region of figure 8 , we may perturb $g$ to eliminate all $p / q$-periodic points (except $z_{0}$ ) in this region. As above, we may perturb $g$ away from $z_{0}$ to eliminate any remaining $p / q$-periodics and we have an area-preserving twist map with only one $p / q$-periodic orbit. This contradicts Birkhoff's theorem.

Hence we see that in any case the set of parameter values for which $f_{s}$ has a $p / q$-monotone periodic point can have no boundary points, so the original map $f$ must have a $p / q$-monotone periodic point, thus completing the proof.

Remark. The technicalities of the last four paragraphs can be avoided if we take a 'generic' one-parameter family of Hamiltonians to produce the one-parameter family of maps $f_{s}$. By 'generic' we mean that each $f_{s}$ has no more than one degenerate $p / q$-periodic point. Since theorem 1 implies that each $f_{s}$ must have at least two monotone $p / q$-periodic points, each $f_{s}$ would have to have at least one non- 


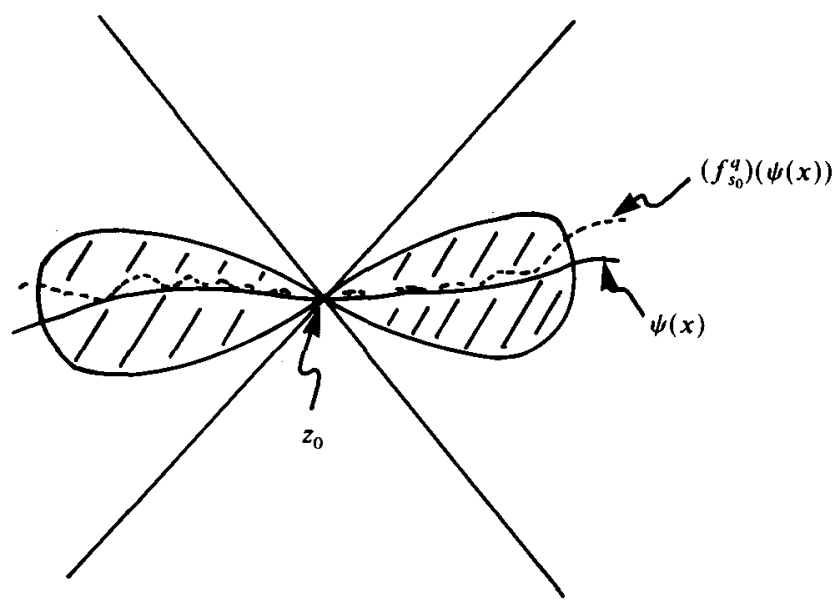

FIGURE 8

degenerate, hence structurally stable, $p / q$-monotone periodic point. See [1] for the technicalities of producing such a generic family.

Acknowledgements. The philosophy and many of the techniques behind the approach to twist maps exploited here were learned by the author from Charles Conley, a debt gratefully acknowledged. Thanks are due to all of those who listened and offered suggestions, Phil Boyland, Kevin Hockett, Joel Robbin, and particularly the referee who pointed out an error in the last section along with other helpful comments. This work was supported in part by NSF grant DMS-8506861.

\section{REFERENCES}

[1] R. Abraham \& J. Robbin. Transversal Mappings and Flows. Benjamin, New York, Amsterdam (1967).

[2] D. Asimov \& J. Franks. Unremovable periodic orbits. Preprint.

[3] S. Aubry \& P. Y. Le Daeron. The discrete Frenkel-Kontrova model and its extensions I. Physica $8 D(1983), 381-422$.

[4] G. Birkhoff. Proof of Poincaré's last geometric theorem. Trans. AMS 14 (1913). Also: G. Birkhoff. Collected Mathematical Papers 1. Dover, New York (1968), 673.

[5] G. Birkhoff. An extension of Poincaré's last geometric theorem. Acta Math. 47 (1925). Also: G. Birkhoff. Collected Mathematical Papers 2. Dover, New York (1968), 252.

[6] C. Conley. Isolated Invariant Sets and the Morse Index. CBMS 38. AMS, Providence (1978).

[7] C. Conley \& E. Zehnder. The Birkhoff-Lewis fixed point theorem and a conjecture of V. I. Arnol'd. Invent. Math. 73 (1983), 33-49.

[8] G. Hall. A topological version of a theorem of Mather on twist maps. Ergod. Th. \& Dynam. Sys. 4 (1984), 585-603.

[9] M. R. Herman. Sur les courbes invariantes par les difféomorphismes de l'anneau. Asterisque 1 (1983), 103-104.

[10] A. Katok. Some remarks on Birkhoff and Mather twist map theorems. Ergod. Th. \& Dynam. Sys. 2 (1982), 185-192.

[11] J. Mather. Existence of quasi-periodic orbits for twist homeomorphisms. Topology 21 (1982), 457-467.

[12] J. Moser. Monotone twist mappings and the calculus of variations. Preprint. 